\title{
Expression of parasite virulence at different host population densities under natural conditions
}

\author{
Annette Bieger · Dieter Ebert
}

Received: 18 October 2008/Accepted: 8 January 2009/Published online: 14 February 2009

(C) Springer-Verlag 2009

\begin{abstract}
It has recently been suggested that the expression of parasite virulence depends on host population density, such that infected hosts have a higher sensitivity to density, and thus reach their carrying capacity earlier than uninfected hosts. In this scenario, parasite-induced reduction in fitness (i.e., virulence) increases with host density. We tested this hypothesis experimentally, using outdoor mesocosm populations of Daphnia magna infected by the microsporidian Octosporea bayeri. Contrary to the prediction, virulence was independent of host density. In a competition experiment with initial prevalence of $50 \%$, $O$. bayeri reduced the competitive ability of infected Daphnia within the asexual growth phase independent of initial host population density. In an additional experiment we set up populations with $100 \%$ and $0 \%$ prevalence and followed their population dynamics over the whole season. Consistent with the competition experiment, we found no difference in population dynamics within the asexual growth phase of the host, suggesting that infected hosts are not more sensitive to density than uninfected hosts. The additional experiment, however, included more than the initial growth phase as did the competition experiment. Eventually, after 100 days, $100 \%$ infected populations assumed a reduced carrying capacity compared to uninfected populations. We identify and discuss three reasons
\end{abstract}

Communicated by R. Brandl.

A. Bieger $(\bowtie) \cdot$ D. Ebert

Zoologisches Institut, Universität Basel,

Vesalgasse 1, 4051 Basel, Switzerland

e-mail: annettebieger@gmx.ch

A. Bieger - D. Ebert

Tvärminne Zoological Station,

10900 Hanko, Finland for the discrepancy between our experiment and the predictions.

Keywords Context-dependent virulence . Competition experiment - Daphnia magna . Octosporea bayeri

\section{Introduction}

In host-parasite interactions, the harm done to the host is important for the evolution of both the host and the parasite (Anderson 1982a; Ewald 1983; Read 1994; Ebert and Hamilton 1996). Hosts evolve to increase their fitness by minimizing the negative impact of the parasite, and parasites try to maximize the benefit they obtain from their hosts. The negative impact of the parasite on the host is defined as virulence and may be expressed as a reduction in fecundity or survival of infected hosts as compared to uninfected hosts. Studies on virulence have implications for numerous fields, including medicine, epidemiology, evolution of cooperation, and the evolution of genetic recombination and maintenance of genetic polymorphism. The expression of virulence is believed to be either an adaptive parasite strategy (Ewald 1983; May and Anderson 1983; Mackinnon et al. 2002), an adaptive host response, or a combination of the two (Ebert 1994; Perlmann and Jaenike 2003; Day and Burns 2003). Virulence is often considered a fixed property of host-parasite interactions (Ewald 1983; Bull 1994).

A growing body of studies suggests that virulence is context dependent (Anderson and May 1981; Brown et al. 2000; Ebert et al. 2004; Jokela et al. 2005), for example, that it may depend on the prevalence of the parasite in the population (Bedhomme et al. 2005) or on host condition 
(Brown et al. 2000). Infected hosts may experience higher virulence under stressful conditions, such as high population density and food shortage, than under good conditions. Lively (2006) suggested that the expression of virulence depends on host population density. He modeled the growth of infected populations for an annual asexual host with non-overlapping generations, in which the parasite did not affect mortality but decreased offspring production. He assumed a higher "sensitivity" to density for infected individuals, i.e. that infected hosts suffer from a comparably stronger decrease in birth rate than uninfected hosts as density increases. This resulted in a lower carrying capacity for infected than for uninfected hosts, as is predicted from other models in which infection reduces the number of hosts in a population (Anderson 1982b) and agreed with experimental data (Ebert et al. 2000). Lively (2006) observed that the decrease in fecundity of infected hosts relative to uninfected hosts increases with increasing population density and is highest at the realized carrying capacity in populations of both infected and uninfected hosts. Virulence at equilibrium still increases as the percentage of uninfected individuals increases (because the carrying capacity for uninfected hosts is higher). Relative fecundity is used as a measure of virulence.

Indirect support for Lively's model (2006) comes from a few empirical studies. In a non-manipulative study on tropical trees, fungicide-treated seedlings survive better than untreated seedlings at high density, but not at low density (Bell et al. 2006). Work on bumblebees (Brown et al. 2000) suggests that hosts in better conditions are able to compensate for the effect of infection by enhanced resource consumption. In another study, Bedhomme et al. (2005) found that parasitism can alter intraspecific competition. Infected hosts suffer from a higher cost of parasitism when raised with an uninfected conspecific than with an infected conspecific. Virulence depends on the infection status of the co-occurring hosts. Pulkkinen and Ebert (2004) worked with a microsporidian in aquatic invertebrates and found that in a population at carrying capacity, a sudden drop of resource level (food shortage) leads to a higher mortality of infected hosts relative to uninfected hosts. Infected hosts are apparently more sensitive to the increased stress caused by less food. Altogether these studies indicate that the expression of virulence may be influenced by host condition which depends on the environment.

The above-mentioned studies measured virulence (or surrogates of it) as a reduction in host performance. Although this approach for understanding the evolution of the parasite has limitations [e.g., not every host fitness component is also a parasite fitness component, see Ebert and Bull (2008) for a discussion], it is the preferred method for studying the evolutionary ecology of the host. To study context-dependent virulence, one must estimate virulence under the relevant conditions; for examining density dependence, thus, one must estimate virulence in populations with different densities.

We did mesocosm experiments in a natural Daphnia magna metapopulation located in the Tvärminne archipelago in southwest Finland (Hanski and Ranta 1983; Pajunen 1986), where the microsporidian parasite Octosporea bayeri is common (Ebert et al. 2001). The D. magna-O. bayeri system has features that allow a quantitative assessment of Lively's virulence model (2006). First, the host D. magna reproduces asexually during the planktonic growth phase, so that parasite-host genotype interactions can be controlled. O. bayeri is known to reduce the asexual birthrate of its host. Second, in the exclusion of horizontal transmission, host and parasite fitness are tightly linked due to the perfect vertical transmission of $O$. bayeri (Vizoso et al. 2005). Competitive ability is therefore a suitable virulence measurement and a good approximation of relative fecundity as used by Lively (2006). Third, in the early growth phase of the host, horizontal transmission of the parasite can be excluded. Horizontal transmission requires the accumulation of spores in the aging host followed by host death, which is unlikely in the first weeks of exponential host population growth. Finally, the system allowed us to test for virulence under natural conditions on a population level. In the Daphnia metapopulation, host population density varies naturally both within and between populations over several orders of magnitude.

To test for density-dependent expression of virulence at different initial host densities, we conducted a competition experiment using the Daphnia-Octosporea system. We quantified the short-term effect of the parasite on the competitive ability of the Daphnia host at four different densities. We conducted an additional population experiment to test Lively's (2006) prediction of reduced carrying capacity in infected hosts. We tested whether populations with $100 \%$ infection rates differed from uninfected populations in carrying capacity over a whole season.

\section{Materials and methods}

The host-parasite system

We studied the planktonic crustacean Daphnia magna Strauss, which inhabit freshwater rock pools along the coast of the Baltic sea (Ranta 1979; Pajunen and Pajunen 2003). In the Tvärminne archipelago in southwest Finland, D. magna is the most common Daphnia species, occurring in about $17 \%$ of all rock pools (Pajunen and Pajunen 2003). Daphnia reproduce by means of cyclical parthenogenesis. 
In May, they hatch from resting eggs and generally reproduce asexually throughout most of the season. An adult female asexually produces clutches of up to $100 \mathrm{eggs}$ every 3-4 days at $20^{\circ} \mathrm{C}$. Reproduction starts at the age of about 10 days; the lifespan of $D$. magna reaches up to 60 days under laboratory conditions (Ebert 2005). Depending on conditions (e.g. crowding) males are produced and sexual reproduction is induced. Resting eggs (ephippia), the product of sexual reproduction, undergo diapause during winter or drought periods (Ebert 2005). In the metapopulation, there is a broad range of initial host population densities. Initial population densities are determined by the number of Daphnia that hatch from the resting stages of the seed bank in the sediment of a pool after a drought period or winter. In an extreme case, initial densities may be very low, as new pools are usually colonized by a single or a few foundresses via dispersing stages (Haag et al. 2006).

The microsporidian parasite Octosporea bayeri Jírovec is specific for its host D. magna. It is the most common parasite in this metapopulation, occurring in $45 \%$ of all D. magna populations (Ebert et al. 2001). In previous studies, the parasite has been shown to be locally adapted to its host (Altermatt et al. 2007). It has a direct life cycle and is transmitted vertically to clonal offspring with $100 \%$ fidelity (Vizoso et al. 2005) and horizontally via waterborne spores, released into the water when dead hosts decay (Vizoso et al. 2005). In May, prevalence in an infected population is usually between 20 and $70 \%$ but may reach $100 \%$ in summer (Lass and Ebert 2006).

\section{Experiment 1: prevalence change at different densities}

To estimate virulence at different densities, we set up an experiment to measure the competitive ability of infected Daphnia relative to uninfected Daphnia. Experimental populations were established at four different population densities with an initial prevalence of $50 \%$. After 4 weeks of asexual reproduction, we estimated the change in prevalence. Because in our experiment the parasite transmits vertically at $100 \%$ efficiency and without horizontal transmission, the host's competitive ability and parasite fitness are tightly linked to each other. With perfect vertical transmission, no horizontal transmission, and asexual host reproduction the parasite behaves like a deleterious gene. Host success can thus be used as a measure for parasite success and change in prevalence to calculate the competitive ability of infected hosts compared to uninfected hosts. In populations with overlapping generations, such as in D. magna populations, competitive ability is a better measure for fitness than only growth rates (Hartl and Clark 1997). We therefore used competitive ability in all analyses (see also Altermatt and Ebert 2007).

\section{Sampling and setup}

In early May 2007, we collected more than 300 adult, eggbearing $D$. magna females from one natural rock pool population. This pool is known to have had a D. magna population for 18 years and to have harbored $O$. bayeri for at least 10 years. Each female was genetically unique, having hatched from a sexually produced resting egg. We kept the adult females in the laboratory under good conditions in artificial medium (Klüttgen et al. 1994; modified after Ebert et al. 1998) and with a constant supply of chemostat-grown green algae Scenedesmus sp. Females released offspring within 1-3 days. We determined the infection status of each isofemale line by inspecting wetmount preparations of the mothers for spores of $O$. bayeri under a microscope (phase-contrast, $400 \times$ magnification). Because O. bayeri transmission is $100 \%$ during asexual reproduction, the newborn could thus be classified as infected or uninfected and used in the field experiment at the age of 3-4 days. We used synchronously hatched juveniles to form the experimental populations. All individuals experienced less than a week in the laboratory.

Between May 16 and 21, we placed 48 experimental populations in plastic buckets filled with 61 filtered $(20 \mu \mathrm{m}$ mesh size) natural pool water next to natural rock pools. The populations were set up with $50 \%$ naturally infected juveniles and $50 \%$ uninfected juveniles. They started at four different densities of 16, 32, 64 and 256 individuals per bucket; these contained 2.5, 5, 10 and 40 Daphnia $1^{-1}$ respectively. Our original plan to include a treatment with 128 individuals per bucket had to be discarded due to a shortage of juveniles. This set of four was replicated 12 times with different females as sources for offspring. In this way all replicates had a new set of genotypes, but the same initial genotypic diversity of 16 clones. In all replicates, the same number of offspring per clone $(1,2,4$ and 16 juveniles of eight infected and eight uninfected mothers) were used for each density treatment.

\section{Onset of asexual reproduction}

All populations were monitored for the appearance of the first eggs in the brood chambers; a few females with asexual eggs were detected 8 days after the start. Females with eggs or clutches in their brood pouch were counted in all replicates 11 days after the start of the experiment. No offspring had been released by then. The timespan from when eggs were laid in the brood chamber to the release of a clutch was longer than 5 days, as water temperature was only around $15^{\circ} \mathrm{C}$. We counted the number of females with eggs in the brood pouch non-invasively, which is easily possible by eye. Additionally, we recorded population sizes of the lower densities by counting all individuals in the 
buckets. We estimated population sizes in the higher densities by taking the mean of three volumetric samples. Individuals per volumetric sample were counted at site and placed back into the buckets. Using this information we estimated the proportion of females carrying clutches.

\section{Prevalence estimation}

4 weeks after the start of the experiment, random samples from each bucket were brought into the laboratory. At this time, the founder females were still present together with the offspring of their first two to four clutches. Offspring of the first clutch were about to reproduce. Thus, the animals spent about 1.5 generations in the experimental conditions. From each replicate population approximately 50 individuals were kept for at least 10 days under laboratory conditions (with constant food supply and in low densities) to ensure that parasites developed sufficiently to allow for spore detection. All host individuals were inspected for infection under a microscope (phase-contrast, 400× magnification). Host sex was recorded as well.

\section{Competitive ability}

We calculated competitive ability (c) of infected hosts relative to uninfected hosts in the populations as a function of change in frequency during clonal growth (Hartl and Clark 1997): $\quad \mathrm{t} * \ln (\mathrm{c})=\ln \left(\mathrm{I}_{\mathrm{t}} / \mathrm{U}_{\mathrm{t}}\right)+\ln \left(\mathrm{I}_{0} / \mathrm{U}_{0}\right), \quad$ where $I_{t}$ and $I_{0}, U_{t}$ and $U_{0}$ represent the frequencies of infected and uninfected female clones at times $t$ and 0 , respectively. Since the initial frequencies were $50 \%, \ln \left(I_{0} / \mathrm{U}_{0}\right)$ equals 0 in our case. For simplicity, $t$ was set to 1 in the analysis.

\section{Statistics}

Prevalence of $O$. bayeri in the experimental populations was tested for an effect of initial host population density. Initial population density was $\log _{10}$ transformed to obtain a stepwise duplication of the density treatments. We analyzed the binomial infection data using a generalized linear model (GLM) with a logit link function (logistic regression) and specified it for a quasi-binomial error distribution because of over-dispersion. All analyses were performed in R (R Development Core Team 2006) using the libraries MASS and Hmisc.

Experiment 2: density dynamics in infected and uninfected populations

In this experiment we followed population dynamics in replicates with prevalences of either $0 \%$ or $100 \%$ at both low and high initial density over 103 days. The time-span of 103 days is comparable to about $80 \%$ of the Daphnia growth season in our study region and reflects about five asexual generations of the host. We measured peak and final host population densities to compare infected with uninfected populations under conditions of initially low and high host density.

\section{Setup and density estimation}

We obtained infected and uninfected offspring as in experiment 1 . Juveniles were hatched in the laboratory, and at 3-4 days old were placed in the outdoor experiment. Populations with prevalences of $0 \%$ and $100 \%$ in high (10 Daphnia $\left.1^{-1}\right)$ and in low densities (2.5 Daphnia $1^{-1}$ ) were set up in plastic buckets filled with 61 of filtered (20 $\mu \mathrm{m}$ mesh size) natural pool water. Eight infected isofemale lines (female clone lineages) were used in the low and the high initial density treatment, and other eight uninfected isofemale lines were used for the uninfected high and low density treatments. Each combination was repeated 6 times using different isofemale lines (infected populations, $6 \times 2$ densities; uninfected populations, $6 \times 2$ densities). Populations were placed next to natural rock pools on 14 May. During the growth phase of the populations (day 0 until day 34) we took non-invasive density samples (at least three independent volumetric samples of $250 \mathrm{ml}$ ) every 3 days, counted individuals directly at site and placed them back into the buckets. Afterwards, density samples (three independent volumetric samples of $100 \mathrm{ml}$ ) were taken approximately every 10 days. After 103 days, on 26 August, all populations were sampled destructively and the total number of Daphnia was counted.

\section{Statistics}

The maximal population densities reached in each bucket were analyzed using a two-way factorial ANOVA, with infection and initial population density as fixed factors. The same was done for the densities on the last sampling day. Two control populations in the highest density experiment had to be excluded because of accidental parasite infection.

\section{Results}

Experiment 1: prevalence change at different densities

Eleven days after the onset of the experiment (age of Daphnia $=15$ days), the estimated mean population sizes per 6-1 bucket were $15.3( \pm 0.3 \mathrm{SE}), 30.2$ ( $\pm 0.5 \mathrm{SE}), 69.0$ $( \pm 4.8 \mathrm{SE})$ and $189.3( \pm 7.6 \mathrm{SE})$ individuals, in the lowest to the highest density treatment respectively. Without mortality we would expect to find 16, 32, 64 and 256 
individuals, the same numbers as initially placed into the buckets. In the highest density treatment, the observed population sizes were lower than expected ( $t$-test, $t=$ $-8.8, P<0.001)$, indicating density-dependent mortality among the founder juveniles.

A density effect was also visible in the form of delayed asexual reproduction. With increasing initial density, a smaller proportion of females was able to start asexual reproduction before the age of 15 days [linear regression on proportions (arc-sine square-root transformed); initial density ( $\log _{10}$ transformed): $t=-5.3, P<0.001$; Fig. 1].

\section{Prevalence}

Initial parasite prevalence was $50 \%$. Mean prevalence of $O$. bayeri across all density treatments was significantly lower than the initial $50 \%$ after 4 weeks of competition (global mean of prevalence in females $=38 \% \pm 2 \%$, proportion test; $\chi^{2}=65.11, d f=1, P<0.001 ; n=48$ ), indicating that infected female Daphnia have a reduced fecundity or survival. This reduction was independent of the initial population density [GLM with quasi-binomial distribution and a logit link function; initial density $\left(\log _{10}\right.$ transformed): $t=-1.24, P=0.22 ; n=48$; Fig. 2a]. The results did not change when initial densities were not log transformed. The reduced prevalence translates (via transformation) into a reduction in competitive ability of $26 \%$ relative to uninfected females across all experimental populations. Further analysis revealed that this effect was somewhat dependent on the sex of the offspring. D. magna infected with $O$. bayeri are known to produce more male offspring than uninfected mothers (Roth et al. 2008). In

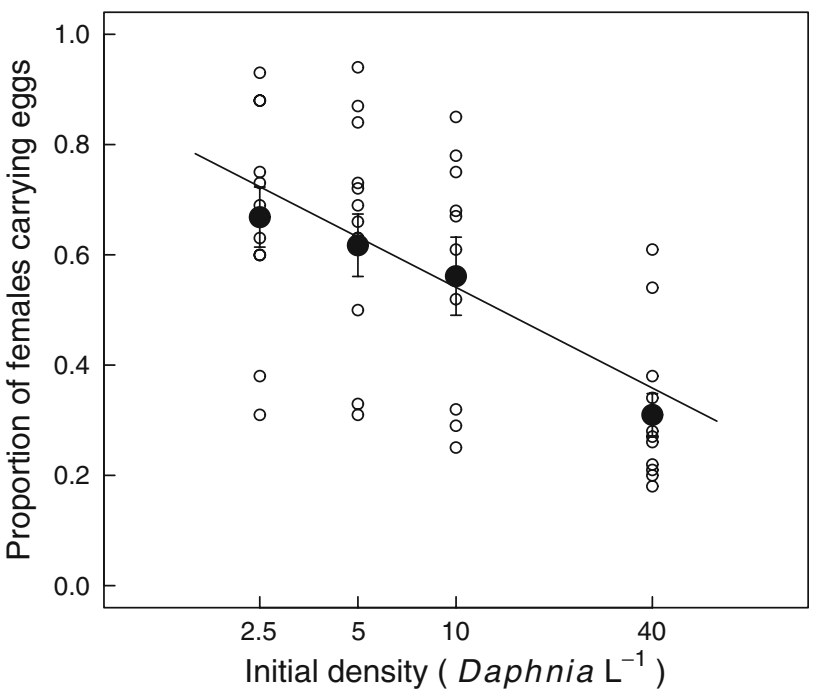

Fig. 1 Percentage of females carrying their first clutch (measured 11 days after onset of the experiment). There is a significant decrease in the proportion of egg-carrying females as initial population density increases (depicted on a $\log _{10}$ scale) addition, no significant density dependence was revealed when we included males in the prevalence and competitive-ability estimates [GLM with quasi-binomial distribution and a logit link function; initial density $\left(\log _{10}\right.$ transformed): $t=-1.49, P=0.14 ; n=48$; Fig. 2c, d]. Prevalence over all treatments was still significantly lower than $50 \%$ (global mean of prevalence $=45 \% \pm 2 \%$, proportion test; $\chi^{2}=22.69, \quad d f=1, \quad P<0.001 ; n=48$; Fig. 2c).

Experiment 2: population dynamics in infected and uninfected populations

We monitored population dynamics in populations with prevalences of either $0 \%$ or $100 \%$ and initial densities of 2.5 or 10 Daphnia $1^{-1}$. All populations went through a phase of exponential growth, reached a peak and then smoothly resumed their carrying capacity (Fig. 3). Peak population densities were reached by 28 and 34 days after the onset of the experiment. They did not differ between infected and uninfected populations and did not significantly depend on initial population density (two-way ANOVA on peak densities; infection, $F=0.60, P=0.45$; initial density, $F=3.09, P=0.10 ; n=22$; Fig. 3 ). At the end of the experiment, infected populations had on average of 50\% less animals than uninfected populations (two-way ANOVA on final densities; infection, $F=30.24, P<0.001$; initial density, $F=3.83, P=0.07$; infection $\times$ initial density, $F=3.10, P=0.10 ; n=22$; Fig. 3). Populations with low initial density reached only a marginally higher final density than populations with high initial density.

\section{Discussion}

Lively (2006) suggests that virulence, measured as competitive ability in a population context, is strongly dependent on density. He postulates that as population density increases, the birth rate of infected hosts decreases more quickly than the birth rate of uninfected hosts. As a consequence, infected hosts reach their carrying capacity at a time when uninfected hosts are still in their growth phase.

We used the Daphnia-Octosporea system to test for a relationship between virulence, estimated as parasitereduced competitive ability, and host population density. Contrary to the prediction, we found that the endoparasite $O$. bayeri influenced its host competitive ability independently of initial host population density (Fig. 2).

In our metapopulation system, host densities between rock pool populations vary strongly in spring, and the system seems very suitable to test for density-related effects. Contrary to our expectations, we found no densitydependent virulence within the first month of exponential 


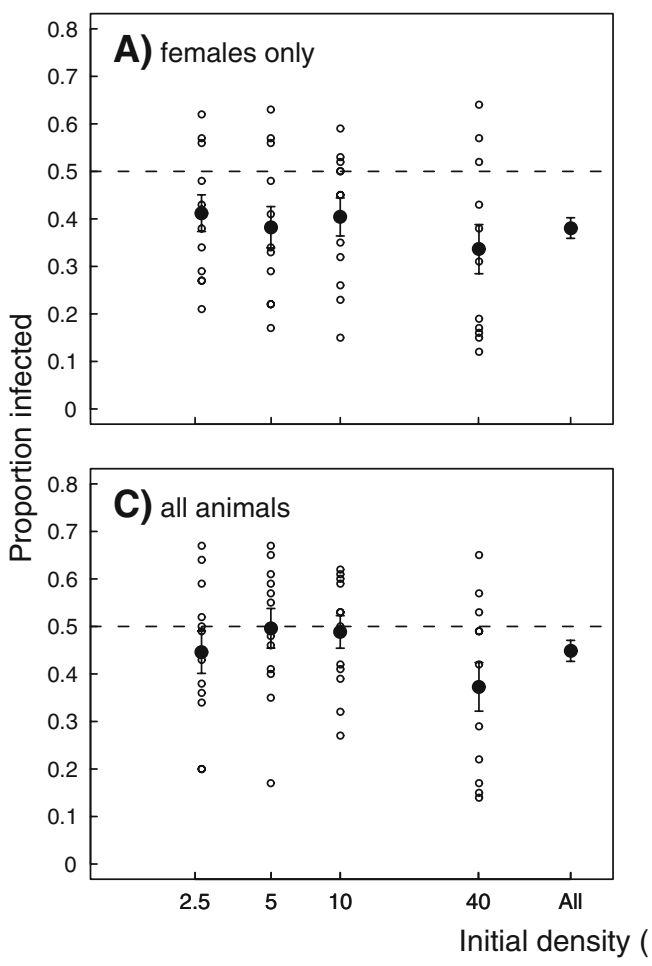

Fig. 2 a Prevalence of Octosporea bayeri in Daphnia magna populations with differing initial densities. Populations were set up with $50 \%$ infected females, and prevalence was estimated in females after 4 weeks. Initial population densities are depicted on a $\log _{10}$ scale; filled circles indicate the means $( \pm \mathrm{SEM})$, and the dotted line refers to a prevalence of $50 \%$. b Relative competitive ability of infected female Daphnia after 4 weeks of clonal growth. Uninfected females are set to a competitive ability of 1 . Depicted are the

host growth (Fig. 2). Our experiment started with densities ranging over a 16 -fold difference from the lowest to the highest density. One may argue that we have not covered the whole range of possible initial densities, and, as a consequence, our treatment may have not produced enough variance with regard to parasitism and asexual reproduction to see the predicted effect. However, we think that this is unlikely. Our lowest density of 2.5 animals $1^{-1}$ water is rather low; lower densities occur in nature only after founder events (D. Ebert, unpublished observation). The rapid density increase over more than 2 orders of magnitude (Fig. 3) clearly indicates that we started far below the carrying capacity. Furthermore, across the density range used, we saw clear signs of density-dependent life history shifts (Fig. 1). Using a larger density range would stretch the range into extreme conditions, which may not be typical in nature. We therefore believe that our treatments were appropriate to test Lively's hypothesis in this system.

A careful analysis revealed three factors that may explain the apparent discrepancy between the model's predictions and our experimental results.

First, host resources, which are used by the parasite, may become limited when the host is under stressful

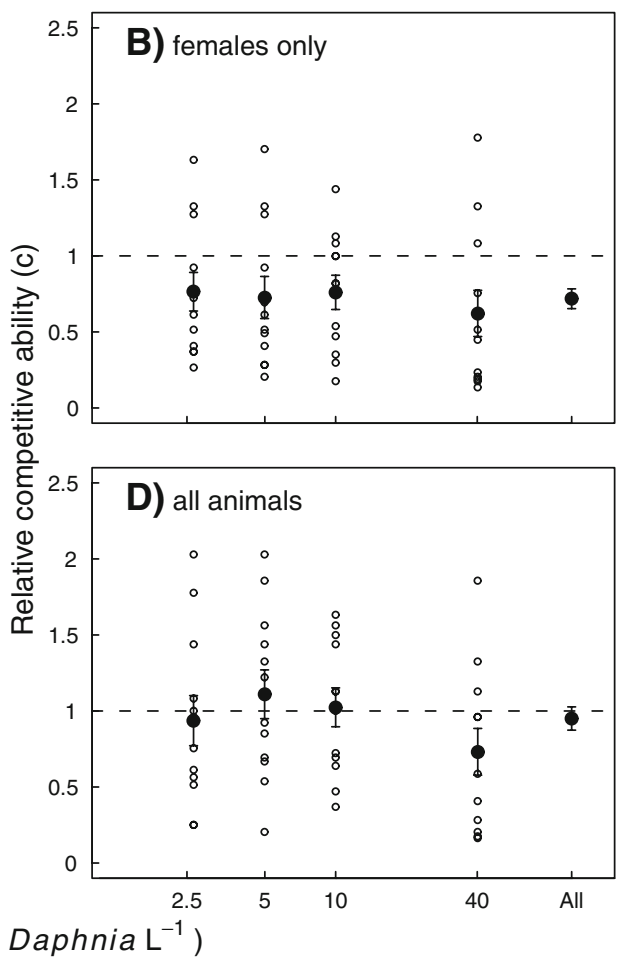

calculated competitive abilities for each density treatment and the overall mean $( \pm$ SEM). Relative competitive ability equals the ratio of growth rates in infected and uninfected hosts; a value of 1 indicates no difference in competitive ability. Across all densities, the competitive ability of infected females is reduced by $26 \%$. c As a, but including all (female and male) Daphnia. d As b, but competitive ability calculated including females and males. Note that competitive ability of infected individuals is only $5 \%$ reduced overall

conditions, such as high density and food shortage. Thus, parasite exploitation may decrease with deteriorating host quality, as was suggested by other studies on invertebrates (Ebert et al. 2000; Jokela et al. 2005; Seppälä et al. 2008). Seppälä et al. (2008) showed that deteriorating conditions for a snail host limit the amount of resources available for the parasite. Ebert et al. (2004) showed that the within-host growth rate of the parasite Pasteuria ramosa in D. magna was lower under low food conditions than under high food conditions. If our high initial density treatment was stressful for both the host and the parasite, virulence may not increase with increasing initial host population density, but the effects on host and parasite may balance each other out, or the parasite may be even less virulent when the host is doing very poorly. Thus, without taking the host density effect on the parasite into account one may potentially reach wrong conclusions.

Second, Lively (2006) models an asexual host population with non-overlapping generations and without recovery. In the model, lifetime fecundity is used as a fitness measure and the parasite reduces host fecundity. Daphnia have overlapping generations, with the female producing several consecutive clutches during her lifetime. 


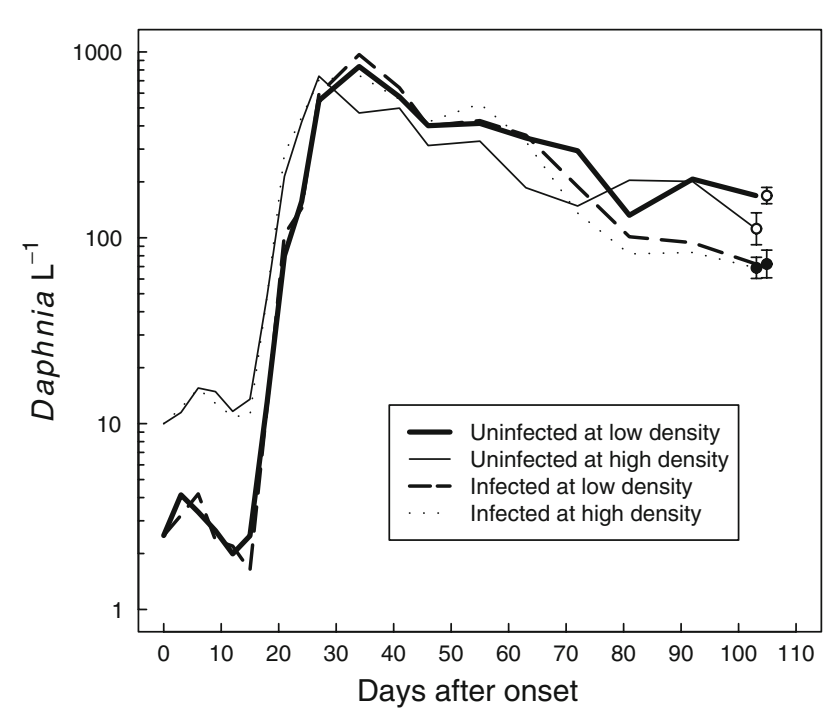

Fig. 3 Average density dynamics in infected and uninfected experimental populations. Infected and uninfected populations started at different densities (2.5 Daphnia $1^{-1}$ (low density) and 10 Daphnia $1^{-1}$ (high density)]. On the last day of monitoring (day 103), populations were fully counted (destructive sampling); the means are depicted $( \pm$ SEM). Peak densities did not differ between the treatments, whereas final densities in infected populations were significantly lower than in uninfected populations. Final densities did not significantly depend on initial population densities. In total 22 populations were used

Until now, all known parasitic diseases of Daphnia are chronic (Ebert 2005), i.e. there is no recovery. Typical for chronic diseases, the effect of the parasite on the host increases the longer it is infected, which usually correlates well with host age (Anderson and Gordon 1982; Hudson and Dobson 1997). In our experimental populations, females released their first clutch 12-16 days after the onset of the experiment and the following three to four clutches within the next two and a half weeks. The negative effect of $O$. bayeri infections on host fecundity increases with host age (Vizoso and Ebert 2005). O. bayeri grows rapidly in infected hosts until it reaches an asymptote, which is a likely indication of resource limitation (Vizoso and Ebert 2004, 2005). The age-dependent decline of fecundity in infected hosts has important consequences for population dynamics. While early reproduction is important during population growth, later clutches contribute little to positive growth but play a role during period of zero or negative growth (Cole 1954; Stearns 1976). In accordance with this, we interpret the lag of a density effect on virulence during early population growth as the result of low fecundity reduction in early clutches (Figs. 2, 3). The $100 \%$ infected populations grew at the same rate and reached the same peak densities as uninfected populations within the same time period (Fig. 3). Positive exponential host growth rates seem to be unaffected by the parasite. Past the peak densities, population densities declined
(Fig. 3), and it is during this phase that late clutches become important (Stearns 1976). Late clutches in D. magna are those most strongly affected by parasitic infections, including $O$. bayeri infections (Carius et al. 2001; Bittner et al. 2002; Vizoso and Ebert 2005). Thus, a second possible reason we could not confirm Lively's (2006) prediction could be because Daphnia populations have overlapping generations, and the parasite effect on fecundity becomes only visible in late clutches. The latter has also been observed for other parasites (Anderson and Gordon 1982; Hudson et al. 1992; Jollens et al. 2005).

Third, we found that competitive ability was significantly reduced during the exponential growth phase when only female Daphnia were considered (competitive ability of infected females: 0.74; Fig. 2b). This reduction was smaller when male and female Daphnia were included in the fitness estimation (competitive ability of infected Daphnia: 0.95; Fig. 2d). We first excluded males because they do not contribute to asexual population growth. Including male offspring in the calculations, however, does not violate the assumption of our competitive ability calculation, as the experiment was too short to include third generation offspring into the experiment (the occurrence of male offspring did not slow down population growth rate at this stage). Thus, with regard to the tested hypothesis, male offspring are as valid as female offspring; both reflect their mother's fecundity, and production costs for sons and daughters do not differ (D. Ebert, unpublished data).

In our second experiment, male offspring may have played a role in reducing population growth rate. Laboratory experiments from the $O$. bayeri system suggest that the production of male offspring is a direct consequence of parasite infections (Roth et al. 2008). Thus, the occurrence of male offspring may have influenced the host population dynamics in the longer-running second experiment. However, this is not the only reason for reduced carrying capacity in infected populations. Daughter production and the survival of infected mothers also declines with age (Vizoso and Ebert 2005; Roth et al. 2008). Sex-specific effects of $O$. bayeri on D. magna go far beyond the assumptions of Lively's model (2006). It seems to interfere with the model's assumptions, but male production alone cannot account for our failure to detect density dependence of virulence.

\section{Conclusion}

The model of Lively (2006) is logical in its construction and appealing in its simplicity. However, we could not confirm its predictions about density-dependent virulence. This failure may be in part system-specific (overlapping vs. non-overlapping generations) or may be more generally 
part of host-parasite interactions (relative fecundity depends on time since infection; parasite success depends on host condition). In host populations with overlapping generations below the carrying capacity, early reproduction is favored; therefore, age structure may be an important factor to include in further models of the density dependence of virulence. A more complex model would lead to a better understanding of the factors shaping the expression of virulence and would allow us to predict under which conditions and in which systems virulence should be density dependent.

Acknowledgements We thank Curt Lively and an anonymous reviewer for their comments improving this manuscript. Thanks to Florian Altermatt and Thomas Zumbrunn for ideas and help during the whole project. We also thank David Duneau and Dita Vizoso for helpful comments on the manuscript and Suzanne Zweizig for improving the English. A. B. was supported by the Werenfels fonds (FAG) and the Anna Caroline Stiftung. The study was supported by the Swiss National Science Foundation. This work is part of project no. 97524006 at Tvärminne Zoological Station. This research was carried out in compliance with the current laws of Finland governing ethical conduct and the use of animals in research.

\section{References}

Altermatt F, Ebert D (2007) Thegenotype specific competitive ability does not correlate with infection in natural Daphnia magna populations. PLoS ONE 2:e1280

Altermatt F, Hottinger J, Ebert D (2007) Parasites promote host gene flow in a metapopulation. Evol Ecol 21:561-575

Anderson RM (1982a) Population dynamics of infectious diseases: theory and applications. Chapman and Hall, London

Anderson RM (1982b) Theoretical basis for the use of pathogens as biological control agents of pest species. Parasitology 84:3-33

Anderson RM, Gordon DM (1982) Processes influencing the distribution of parasite numbers within host populations with special emphasis on parasite-induced host mortalities. Parasitology 85:373-398

Anderson RM, May RM (1981) The population dynamics of microparasites and their invertebrate hosts. Philos Trans R Soc Lond B Biol Sci 291:451-524

Bedhomme S, Agnew P, Vital Y, Sidobre C, Michalakis Y (2005) Prevalence-dependent costs of parasite virulence. PLoS Biol 3:1403-1408

Bell T, Freckelton RP, Lewis OT (2006) Plant pathogens drive density-dependent seedling mortality in a tropical tree. Ecol Lett 9:569-574

Bittner K, Rothaupt KO, Ebert D (2002) Ecological interactions of the microparasite Caullerya mesnili and its host Daphnia galeata. Limnol Oceanogr 47:300-305

Brown M, Loosli R, Schmid-Hempel P (2000) Condition dependent expression of virulence in a Trypanosome infecting bumbelbees. Oikos 91:421-427

Bull JJ (1994) Perspective: virulence. Evolution 48:1423-1437

Carius HJ, Little TJ, Ebert D (2001) Genetic variation in a hostparasite association: potential for coevolution and frequencydependent selection. Evolution 55:911-921

Cole LC (1954) The population consequences of life-history phenomena. Q Rev Biol 29:103-137

Day T, Burns JG (2003) A consideration of patterns of virulence arising from host-parasite co-evolution. Evolution 57:671-676
Ebert D (1994) Virulence and local adaptation of a horizontally transmitted parasite. Science 265:1084-1086

Ebert D (2005) Ecology, epidemiology, and evolution of parasitism in Daphnia. National Library of Medicine (US), National Center for Biotechnology Information, Bethesda. Available from: http://www. ncbi.nlm.nih.gov/entrez/query.fcgi?db=Books

Ebert D, Bull J (2008) The evolution and expression of virulence. In: Stearns SC, Koella JC (eds) Evolution in health and disease. Oxford University Press, Oxford, pp 153-154

Ebert D, Hamilton WD (1996) Sex against virulence: the coevolution of parasitic diseases. Trends Ecol Evol 11:79-81

Ebert D, Zschokke-Rohringer CD, Carius HJ (1998) Within- and between-population variation for resistance of Daphnia magna to the bacterial endoparasite Pasteuria ramosa. Proc R Soc Lond B Biol Sci 265:2127-2134

Ebert D, Lipsitch M, Mangin KL (2000) The effect of parasites on host population density and extinction: experimental epidemiology with Daphnia and six microparasites. Am Nat 156:459-477

Ebert D, Hottinger JW, Pajunen VI (2001) Temporal and spatial dynamics of parasites in a Daphnia metapopulation: which factors explain parasite richness? Ecology 82:3417-3434

Ebert D, Carius HJ, Little T, Decaestecker E (2004) The evolution of virulence when parasites cause host castration and gigantism. Am Nat 164:19-32

Ewald PW (1983) Host-parasite relations, vectors, and the evolution of disease severity. Annu Rev Ecol Syst 14:456-485

Haag CR, Riek M, Hottinger W, Pajunen VI, Ebert D (2006) Founder events as determinants of within-island and among-island genetic structure of Daphnia metapopulations. Heredity 96:150-158

Hartl D, Clark A (1997) Principles of population genetics, 3rd edn. Sinauer, Sunderland

Hanski I, Ranta E (1983) Coexistence in a patchy environment: threespecies of Daphnia in rock pools. J Anim Ecol 52:263-279

Hudson PJ, Dobson AP (1997) Host-parasite processes and demographic consequences. Oxford University Press, New York

Hudson PJ, Newborn D, Dobson AP (1992) Regulation and stability of a free-living host-parasite system: Trichostrongylus tenuis in red grouse. I. Monitoring and parasite reduction experiments. J Anim Ecol 61:477-486

Jokela J, Taskinen J, Mutikainen P, Kopp K (2005) Virulence of parasites in hosts under environmental stress: experiments with anoxia and starvation. Oikos 108:156-164

Jollens AE, Cooper DV, Levin SA (2005) Hidden effects of chronic tuberculosis in African buffalo. Ecology 86:2258-2264

Klüttgen B, Dülmer U, Engels M, Ratte HT (1994) ADaM, an artificial freshwater for the culture of zooplankton. Water Res 28:743-746

Lass S, Ebert D (2006) Apparent seasonality of parasite dynamics: analysis of cyclic prevalence patterns. Proc R Soc Lond B Biol Sci 273:199-206

Lively CM (2006) The ecology of virulence. Ecol Lett 9:1089-1095

Mackinnon MJ, Gaffney D, Read A (2002) Virulence in rodent malaria: host genotype by parasite genotype interactions. Infect Genet Evol 1:287-296

May RM, Anderson RM (1983) Epidemiology and genetics in the coevolution of parasites and hosts. Proc R Soc Lond B Biol Sci 219:281-313

Pajunen VI (1986) Distributional dynamics of Daphnia species in arock-pool environment. Ann Zool Fenn 23:131-140

Pajunen VI, Pajunen I (2003) Long-term dynamics in rock pool Daphnia metapopulations. Ecography 26:731-738

Perlmann SJ, Jaenike J (2003) Evolution of multiple components of virulence in Drosophila-nematode associations. Evolution 57:1543-1551

Pulkkinen K, Ebert D (2004) Host starvation decreases parasite load and mean host size in experimental populations. Ecology 85:823-833 
R Development Core Team (2006) R: a language and environment for statistical computing. R Foundation for Statistical Computing. Vienna

Ranta E (1979) Niche of Daphnia species in rock pools. Arch Hydrobiol 87:205-223

Read AF (1994) The evolution of virulence. Trends Microbiol 2:73-76

Roth O, Ebert D, Vizoso DB, Bieger A, Lass S (2008) Male-biased sex-ratio distortion caused by Octosporea bayeri, a vertically and horizontally-transmitted parasite of Daphnia magna. Int J Parasitol 38:969-979

Seppälä O, Liljeroos K, Karvonen A, Jokela J (2008) Host condition as a constraint for parasite reproduction. Oikos 117:749-753
Stearns SJ (1976) Life-history tactics: a review of the ideas. Q Rev Biol 51:3-47

Vizoso D, Ebert D (2004) Within-host dynamics of a microsporidium with horizontal and vertical transmission: Octosporea bayeri in Daphnia magna. Parasitology 128:31-38

Vizoso D, Ebert D (2005) Phenotypic plasticity of host-parasite interactions in response to the route of infection. $J$ Evol Biol 18:911-921

Vizoso D, Lass S, Ebert D (2005) Different mechanisms of transmission of the microsporidium Octosporea bayeri: a cocktail of solutions for the problem of parasite permanence. Parasitology 130:501-509 